\title{
EEG Signal Classification using Frequency Band Analysis towards Epileptic Seizure Prediction
}

\begin{abstract}
Epilepsy is one of the most common and diverse set of chronic neurological disorders is characterized by an abnormal excessive or synchronous neuronal activity in the brain that is termed "seizure", affecting about 50 million individuals worldwide. Electroencephalogram (EEG) signal processing technique plays a significant role in detection and prediction of epileptic seizures. Recently, lots of research work has been devoted to the prediction of epileptic seizures based on analysis of EEG signals. Even though remarkable worked on seizure prediction, experimental results are not mature enough in terms of sensitivity, specificity, and accuracy for prediction. In this paper we present a new approach for seizure detection and prediction by extracting different features from different frequency bands (e.g., gamma, beta, alpha, theta, and delta) of the EEG signal by decomposing the signal using discrete wavelet transformation. We have observed that among different frequency bands, gamma band carries more distinguishing features. Experimental results demonstrate that the proposed method outperforms the state-of-the-art method in terms of sensitivity, specificity and accuracy to classify seizure EEG signals to the benchmark dataset in different brain locations.
\end{abstract}

\section{Keywords- EEG, DWT, LS-SVM, Seizure, and Epilepsy.}

\section{INTRODUCTION}

Seizure is sudden and intensive, unexplained, unknown and coherent neurological activity in the brain. Epilepsy is another medical condition having spontaneous recurrent seizures [1]. Epilepsy take place when many neurons are excited in the same time due to brain injury or chemical imbalance in the brain that is characterized predominantly by unpredictable interruptions of normal brain function. Epilepsy puts patients in many injuries including high risk of fractures, submersion injuries, burns, motor vehicle accidents and many others. Epilepsy affects approximately $1 \%$ of the world's population [1]. It is highly possible to prevent epilepsy if we can reliably predict the pre-syndrome of the epilepsy with high sensitivity and specificity.

Human brain is an electro-chemical machine, because neurons use chemical reaction to generate electricity. Electroencephalogram (EEG) is the well acceptable and most common diagnosis process for epilepsy and can detect abnormalities in the brain's electrical activity. It is highly possible to predict/detect seizure onset through analysis of the EEG signals. The prediction of Epilepsy is clinically apparent in preictal (i.e., before seizure onset) stage of Epilepsy.
Properly prediction of Epilepsy puts away the patients from fractures, submersion injuries, burns, vehicle accidents, etc. Therefore, it is significant to prevent epilepsy through correctly predict/detect preictal stage to get accurate prediction results.

Many methods have been proposed to predict epileptic seizure by classifying preictal and interictal EEG signals. They have employed univariate techniques [3], eigenspectra of space delay correlation and covariance matrices [4], Hilbert-Huang transform[5], and autoregressive modeling and least-squares parameter estimator [6]. According to classification results of preictal and interictal EEG signals with respect to sensitivity, Chisci et al. [6] proposed technique carries good sensitivity compared to above mentioned techniques [4][5] using dataset [7]. However, Rasekhi et al. [3] got $79.3 \%$ sensitivity for their own dataset. The mentioned techniques [3]-[6] have good sensitivity, however, the specificity and accuracy are not expected level.

Recently, many techniques have been proposed for correct seizure prediction and detection. Among the existing techniques, Bajaj et al.'s [8] technique is newest and preeminent in term of performance and it can also measure non-linear dynamics of the EEG signals in proper way using features i.e. amplitude and frequency modulation bandwidth through empirical mode decomposition (EMD) technique. We have observed that Bajaj et al.'s [8] technique exhibit almost perfect classification accuracy for the small dataset [9] of seizure and non-seizure EEG signals. However, their performance is below the expected results using the large dataset [7] for preictal and interictal EEG signal classifications.

Bajaj et al.'s [8] have decomposed the EEG signal into different intrinsic mode functions (IMFs), where they did not explicitly decompose the signal into different frequencies. However, a number of literature including [10] has decomposed the signal into different frequency bands and then extracted different features from different bands to get band specific features for seizure detection/prediction. It is quite understandable that all bands are not related to seizure. Thus, features from some bands are highly related to the seizure compared to that of other bands.

Moreover, with improvement of technologies and increasing number of quality channels, it is essential to realize patterns that are potentially involve in all the EEG 
channels across a range of temporal scales [4]. Temporal correlation of the EEG signals carries good sensitivity for preictal signal detection which leads towards epileptic seizures prediction.

In this paper, we present novel approach for feature extraction and classification through discrete wavelet transform (DWT) to decompose a signal into different frequency bands such as gamma $(30-60 \mathrm{~Hz})$, beta $(13-30 \mathrm{~Hz})$, alpha $(8-12 \mathrm{~Hz})$, theta $(4-8 \mathrm{~Hz})$, and delta $(0-4 \mathrm{~Hz})$. Among the frequency bands, the gamma band is related to seizure as it carries more information than the other bands. This gamma band is used for feature extraction to exploit temporal correlation of the signals. These features are used as an input to least square support vector machine (LSSVM) for classification of preictal and interictal EEG signals. The experimental results show that newly extracted features provide better classification accuracy compared to the existing state-of-the-art method [8] for the classification of preictal and interictal EEG signals using benchmark large size datasets [7] in different brain locations.

\section{PROPOSED METHOD}

In this paper, we proposed a classification technique using two features namely frequency and amplitude from the gamma band signals through DWT decomposition technique using dataset [7], Epilepsy centre of the University Hospital of Freiburg Germany, considering temporal correlation and then classify preictal and interictal signals using LS-SVM.
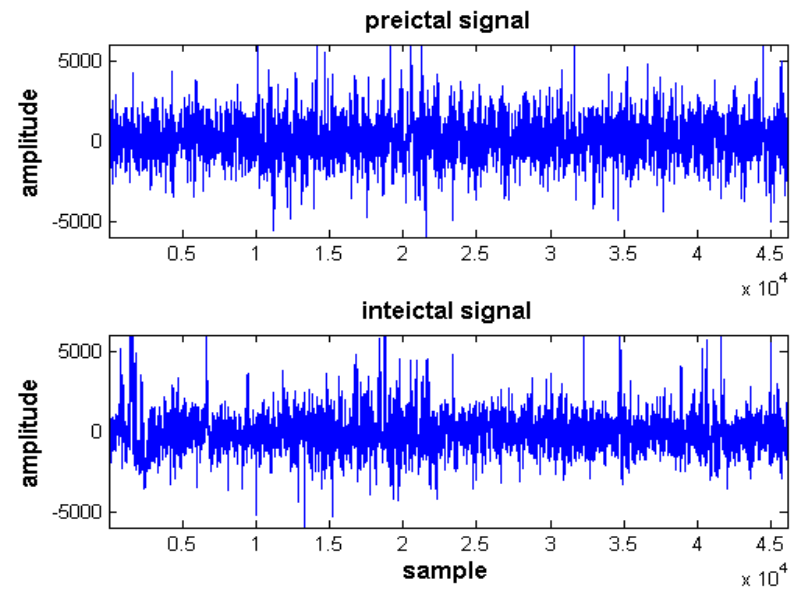

Fig 1: Preictal (patient one, 7th datablock, channel one) and interictal (patient one, 95th datablock, channel one) of Frontal lobe signals [7].

\section{A. Dataset}

The dataset used in the paper was recorded at Epilepsy centre of the University Hospital of Freiburg, Germany [7]. The database contains invasive EEG recordings of 21 patients suffering from medically intractable focal epilepsy. The data obtained by Neurofile NT digital video EEG system with 128 channels, $256 \mathrm{~Hz}$ sampling rate, and 16 bit analogue-to-digital converter. According to the dataset [7], epileptic EEG signals can be classified into (i) ictal (i.e., seizure period), (ii) preictal (before seizure onset), (iii) interictal (period between seizures). Normally, duration of ictal is varied from few seconds to 2 minutes. The ictal records contain epileptic seizures with at least 50 minutes of preictal signal preceding each seizure. The median time period between the last seizure and the interictal signal is 5 hours and 18 minutes, and the median time period between the interictal signal and the first following seizure is 9 hours and 36 minutes [4]. As suggested in the dataset, we have treated preictal as 3-minute prior on seizure onset. To make equal size (i.e., three minutes) of interictal signal with the preictal signal, we also take three minutes from each interictal signal of the dataset. Three minutes are taken from the 7 th minutes of an interictal signal. In this experiment, we have used EEG signals from 12 patients where 6 patients for Frontal lobe signals and 6-patients for Temporal lobe signals. Sample signal is shown in Fig 1.

\section{B. Feature Extraction}

DWT works based on multi-level where each signal decomposed into several scales and each scale provides a particular coarseness of signal. Each phase of decomposition of a signal is composed of two down samplers by 2 and two digital filters. In each phase, high pass filter which serves as the discrete mother wavelet, and low pass filter which acts as the mirror version of the corresponding. The down-sampled outputs of the first low-pass and high-pass filters supply the approximation $A l$ and detail $D l$, respectively. The first approximation $A l$ is further decomposed and the procedure is continued. H. Adeli et al. mentioned that the Daubechies order 4-wavelet $(\mathrm{db} 4)$ is most suitable for EEG signal analysis [13]. In our experiment, we have used Daubechies wavelets $\mathrm{db} 8$ on the right to extract gamma band and then extract features from gamma band to classify preictal and interictal EEG signals.

The wavelet transform can better localize the signal components in time frequency space. The MorletGrossmann defined the continuous wavelet transform for one dimensional $x(t)$ signal is as follows [14]:

$$
W(a, b)=\frac{1}{\sqrt{a}} \int_{-\infty}^{\infty} x(t) \psi^{*}\left(\frac{t-b}{a}\right) d t
$$

where $(.)^{*}$ denoted the complex conjugate, $\psi(t)$ is the analysing wavelet, $a$ is the scalar parameter, and $b$ is the position parameter.

The most common method is to separate continuous wavelet to discrete wavelet by scale factor of binary scales. Let $a=2^{j}$ and the equation is as follows [15]:

$$
W(j, b)=\frac{1}{\sqrt{2^{j}}} \int_{-\infty}^{\infty} x(t) \psi^{*}\left(\frac{t-b}{2^{j}}\right) d t
$$

The first split of signal $x(t)$ is $A l$ and $D l$. Then further $A l$ is split into $A 2$ and $D 2$. This procedure continues until specified level is reached. For example, $A_{i}$ and $D_{i}(i=1$, $2, \ldots, n)$ are the low frequency component and high frequency component of the $i^{\text {th }}$ level respectively. The 4-level DWT is 
shown in Fig 2 and its output signals are shown in Fig 3.

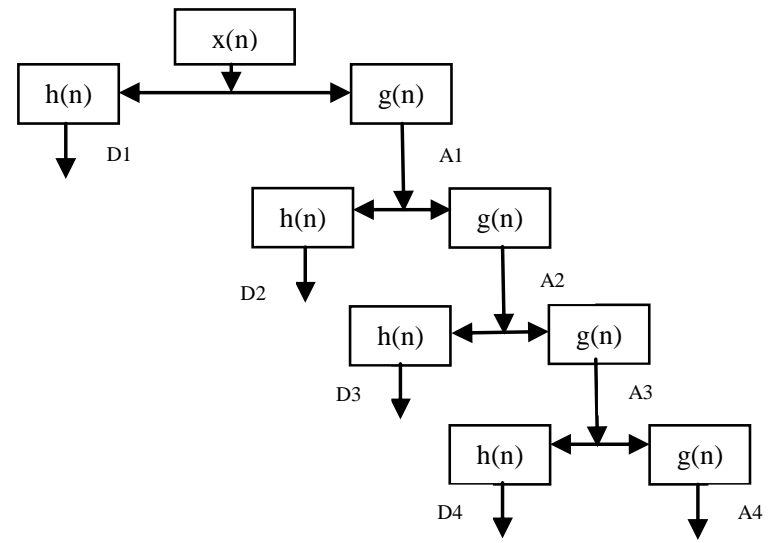

Fig 2: EEG signal decomposition through 4-level discrete wavelet transformation.

To extract the features using DWT, we have calculated gamma band from three minute EEG signals and then reshaped this gamma band into a two dimensional feature vectors to exploit temporal correlation of signals by dividing the signal into 15 seconds epoch (i.e., total 12 epochs for a signal). For gamma band of preictal, the values of frequency distribution is low and amplitude distribution is high whereas the characteristics of interictal signal are reverse as shown in Fig 4. Thus, we consider average of up to 6th minimum frequency and 6th maximum amplitude as features from preictal. For gamma band of interictal, we consider average of up to 6th maximum frequency and 6th minimum amplitude as features. The average values of frequency and amplitude are used as input of the LS-SVM classifier for preictal and interictal classification.
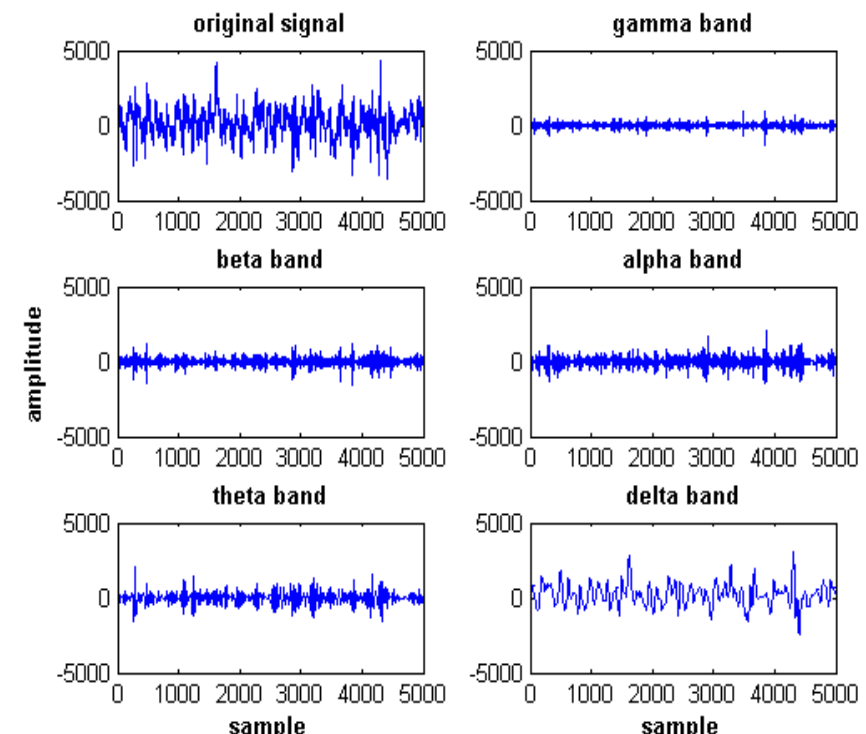

Fig 3: Different frequency band after applying DWT decomposed technique in preictal signal of Frontal lobe signal (patient one, $7^{\text {th }}$ datablock, and channel one).

\section{Classification}

The goal is to find patients states, interictal (Class 1) and preictal (Class 2) using the machine learning approaches through cross-validation evaluation. The challenge is to find the mapping that generalized from training sets and unseen test sets. For the cross-validation, data are partitioned into training set and test set. To classify the ictal and interictal signals, we have extracted features from DCT and IMF. For classification, we have used LS-SVM [11] classifier as the LS-SVM is one of the best classifiers in the EEG signal analysis. It can minimize the operational error and maximize the margin hyperplane, as a result it will maximize the classification performance [12].

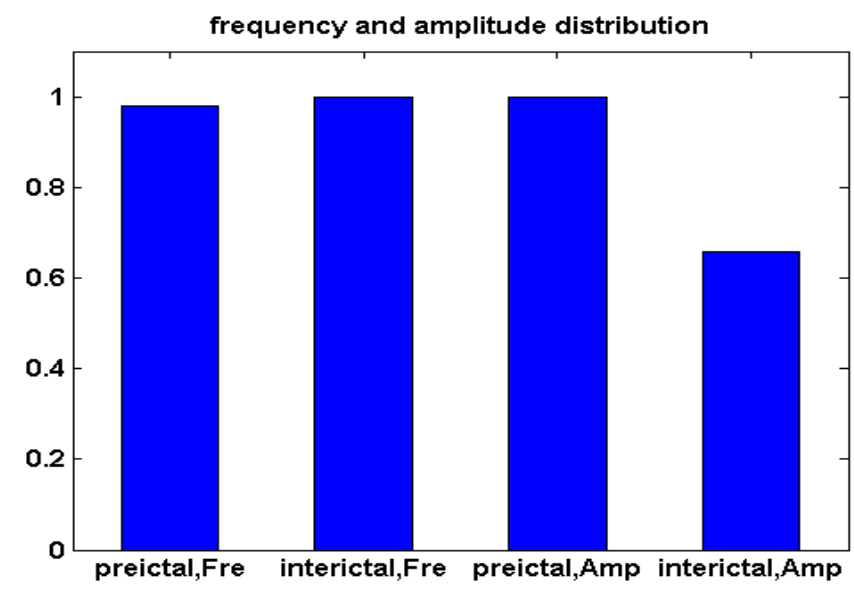

Fig 4: Frequency and amplitude distribution on Frontal lobe EEG signals Preictal signals carries lower and higher distribution for frequency and amplitue, respectivly, whereas interictal signals has reversed order.

Let us consider binary classification problem with the training set $\left\{x_{i}\right\}_{i=1}^{N}$, along with corresponding targets $\left\{y_{i}\right\}_{i=1}^{N}$ where $x_{i} \in R^{n}$ and $y_{i}=\{1,-1\}, i=1, \ldots N$. Assume there exists a linear separable hyperplane $f(w, b)=\|w\|^{2} / 2$ such that

$$
\begin{array}{ll}
w^{T} x_{i}+b \geq 1 & \forall_{i}: y_{i}=1 \\
w^{T} x_{i}+b \leq-1 & \forall_{i}: y_{i}=-1
\end{array}
$$

where the decision function [11] is:

$$
f(x)=\operatorname{sign}\left[w^{T} g(x)+b\right]
$$

where $w$ is the weight vector and $g(x)$ is the mapping function of $x$ and $b$ is a bias.

The optimization problem of LS-SVM can be solved in the following way:

$$
\min _{w, b, \xi} \frac{1}{2}\|w\|^{2}+\frac{C}{2} \sum_{i=1}^{N} \xi_{i}^{2}
$$

subject to $y_{i}\left(\left(w^{T} x_{i}\right)+b\right)=1-\xi_{i}, i=1 \ldots N$.

where $\xi_{i}$ are the slack variables, it represents the upper bound training error and $C>0$ trades off margin size and training error. 
Lagrange multipliers $\alpha_{i}$ can be introducing:

$\min _{w, b, \xi, \alpha} \frac{1}{2}\|w\|^{2}+\frac{C}{2} \sum_{i=1}^{N} \xi_{i}^{2}-\sum_{i-1}^{N} \alpha_{i}\left\{1-y_{i}\left[w^{T} g\left(x_{i}\right)+b\right]-\xi_{i}\right\}$

subject to $\alpha_{i} \geq 0 ; i=1 \ldots N$.

For non-linear problem the kernel function is introduced in the above problem. Therefore, the decision function can be defined:

$$
f(x)=\operatorname{sign}\left[\sum_{i-1}^{N} \alpha_{i} y_{i} K\left(x, x_{i}\right)+b\right]
$$

where $K\left(x, x_{i}\right)$ is a kernel function.

Radial basis function (RBF) kernel is used in our experiments and this function can be defined as:

$$
k\left(x, x_{i}\right)=\exp \left(-\left\|x-x_{i}\right\|^{2} / 2 \sigma^{2}\right)
$$

where $\sigma$ controls the width of the RBF function

\section{EXPERIMENTAL RESULTS}

We have classified the preictal and interictal data extracted from Frontal and Temporal lobe signals using LSSVM classifier with RBF kernel where the value of regularization parameter and kernel parameter are 10 and 6
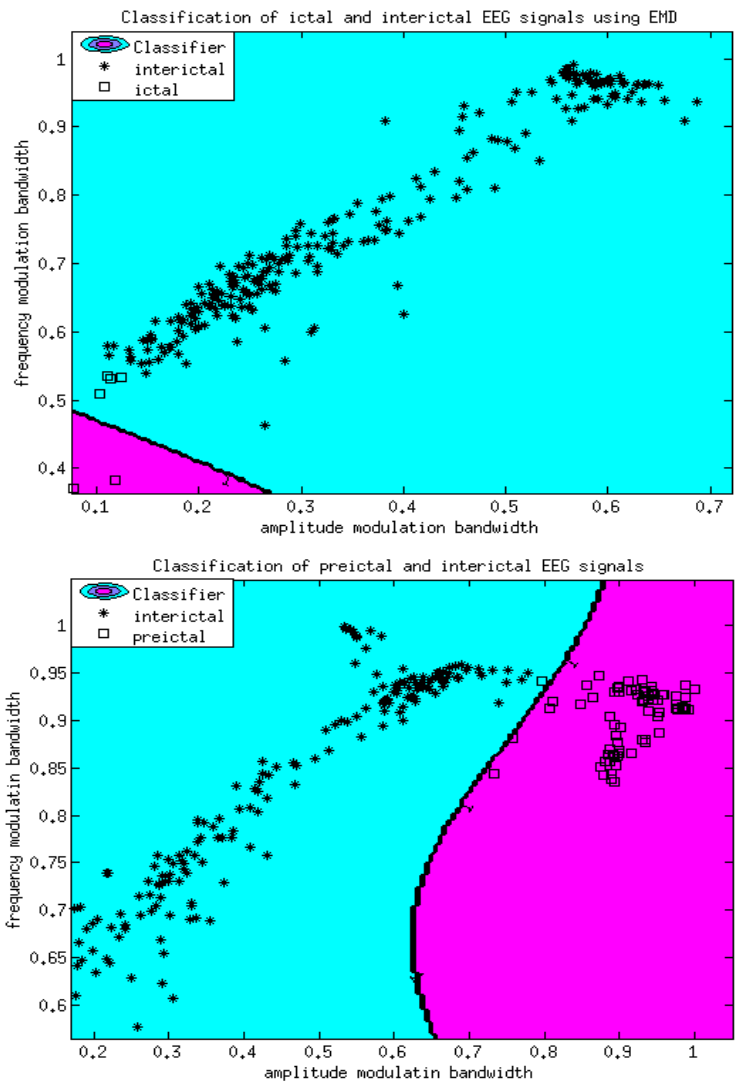

Fig 5: Classification of preictal and interictal EEG signals for seizure prediction. First row represents the classification of preictal and interictal EEG signals from Frontal lobe for the first subset of testing using the state-of-the-art method against the proposed method while second row represents the classification of preictal and interictal EEG signals from Temporal lobe for the third subset of testing using the state-of-the-art method against the proposed method. respectively. In our experiment, we have used 150 signals from preictal data and 882 signals from interictal data of three minutes EEG recording signals from dataset [7] and applied 4-fold cross validation for classification purpose. After feature extraction, we randomly sort the preictal and interictal data for the 1032 signals for each lobe either Frontal or Temporal. For unbiased classification results, we split the whole 1032 signals into four subsets; in each subset we randomly select $75 \%$ samples for the training and the remaining $25 \%$ for the testing. After that, we calculate average value from the four subsets to compute sensitivity, specificity and accuracy.

TABLE 1: SENSITIVITY, SPECIFICITY, AND ACCURACY FOR DIFFERENT FEATURES OF PREICTAL AND INTERICTAL EEG SIGNALS FROM FRONTAL

\begin{tabular}{|l|c|c|cc|}
\hline \multirow{3}{*}{ Kernel } & Lobe & & Existing[8] & proposed \\
& & & & \\
\hline \multirow{4}{*}{ RBF } & \multirow{4}{*}{ Frontal } & SEN & 47.88 & 97.05 \\
\cline { 3 - 3 } & & SPE & 85.67 & 92.73 \\
\cline { 3 - 3 } & \multirow{3}{*}{ Temporal } & ACC & 79.94 & 93.12 \\
\cline { 3 - 3 } & & SEN & 55.20 & 94.00 \\
\cline { 3 - 3 } & & SPE & 86.45 & 93.83 \\
& & ACC & 86.14 & 93.79 \\
\hline
\end{tabular}

It can be observed from Table 1 that the proposed method outperforms the state-of-the-art method [8] in terms of sensitivity, specificity, and accuracy. In terms of three

Classification of preictal and interictal EEG signals from Frontal lobe

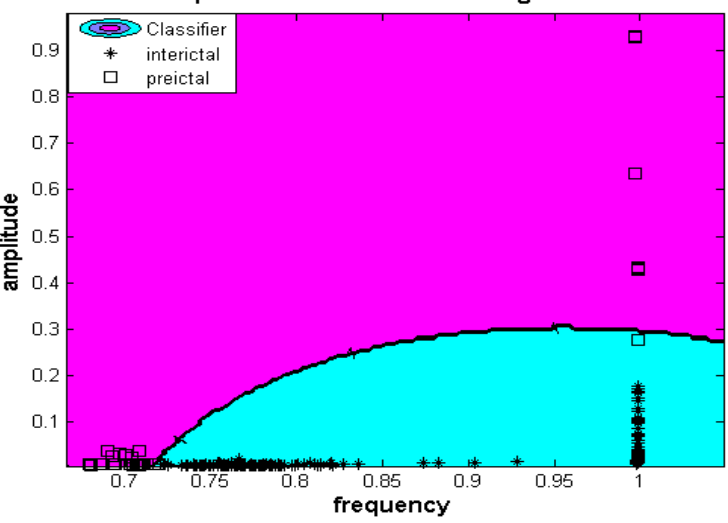

Classification of preictal and interictal EEG signals from Temporal lobe

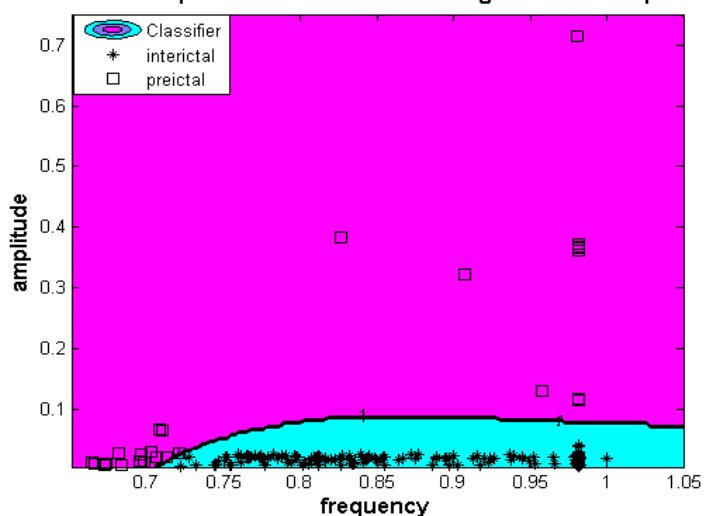


different classification criteria, proposed method performs consistently and the classification performances are $93.12 \%$ and $93.79 \%$ from Frontal lobe and Temporal lobe signals respectively, whereas, the technique [8] does not perform consistently and the classification of performances are $79.94 \%$ and $86.14 \%$ from Frontal lobe and Temporal lobe signals respectively. The classification consistency among three different criteria is very important for proper diagnosis.

Fig 5 shows the visual classification comparison using the proposed methods and the state-of-the-art method [8] for better understanding. We have generated two images in Fig 5 from Frontal lobe for the first subset and the first IMF of testing set and obtained the classification accuracy $81 \%$ by the existing technique [8] (see top left of Fig 5) and 94.18\% by the proposed method (see top right of Fig 5) while stateof-the-art method provide $79.84 \%$ accuracy (see bottom left of Fig 5) and proposed method also provide $95.34 \%$ accuracy (see bottom right of Fig 5)for third subset from Temporal lobe signals. Moreover, visually we can see from Fig 5 that the existing method has more miss-classified preictal signals compared to that of the proposed method. Thus, it can be concluded from Fig 5 that the proposed methods outperform the state-of-the-art method using the dataset in [7].

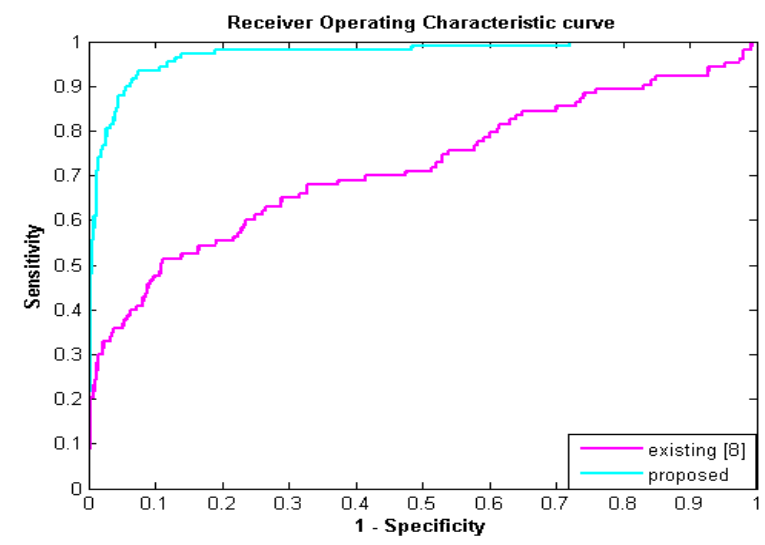

Fig 6: The receiver operating characteristics (ROC) curves of the first subset of training and testing EEG signals by proposed method against the state-of-the-art method using LS-SVM with RBF kernel from Temporal lobe.

The performance of the LS-SVM is evaluated by receiver operating characteristics (ROC) plot is shown in Fig 6. ROC illustrates the performance of a binary classifier system where it is created by plotting the fraction of true positives from the positives i.e., true positive rate (TPR) vs. the fraction of false positives from negatives i.e., false positive rate (FPR) with various threshold settings. TPR is known as sensitivity, and FPR is one minus the specificity or true negative rate. Fig 6 demonstrates that the proposed method is carrying good classification results than that of the state-ofthe-art method [8] using dataset from Temporal lobe of the first subset of training and testing EEG signals.

\section{CONCLUSION}

The progress of a reliable seizure prediction and detection could improve the quality of life of the patients significantly. Hence, it is important to detect forth-coming seizure, not only for patient safety but also to have possibility to stop the ictal event. For this purpose, we need to classify properly preictal and interictal EEG signals with good sensitivity and specificity. In this paper, we present and evaluate a method using DWT that decomposed gamma band by exploiting temporal correlation that is used in intracranial EEG signals to detect the prior stage of seizure onset through LS-SVM classifier. The results show that the proposed method offers a good combination of sensitivity and specificity, thus making the classification of preictal and interictal EEG signals feasible.

\section{REFERENCES}

[1] R. S. Fisher, W. van Emde Boas, W. Blume, C. Elger, P. Genton. P. Lee, and J. Jr. Engel," Epileptic seizures and epilepsy: definitions proposed by the International League Against Epilepsy (ILAE) and the International Bureau for Epilepsy (IBE)," Epilepsia, 46(4), 470-472, 2005.

[2] P. Kwan, and M.J. Brodie," Refractory epilepsy: mechanisms and solutions," Expert Review of Neurotherapeutics, 6(3):397-406, 2006.

[3] J. Rasekhi, M. R. K. Mollaei, M. Bandarabadi, C. A. Teixeira, and A. Dourado," Preprocessing effects of 22 linear univariate features on the performance of seizure prediction methods," Journal of Neuroscience Methods, vol. 217, 9-16, 2013.

[4] J. R. Williamsona, D. W. Blissa, D. W. Brownea, and J. T. Narayananb,” Seizure prediction using EEG spatiotemporal correlation structure," Journal of Epilepsy \& Behavior, vol. 25(2), 230-238, 2012.

[5] F. Duman, N. Ozdemir, and E. Yildirim," Patient Specific Seizure Prediction Algorithm Using HilbertHuang Transform," IEEE-EMBS International Conference on Biomedical and Health Informatics, Hong Kong and Shenzhen, China, 2012.

[6] L. Chisci, A. Mavino, G. Perferi, M. Sciandrone, C. Anile, G. Colicchio, and F. Fuggetta," Real-Time Epileptic Seizure Prediction Using AR Models and Support Vector Machines," IEEE Transactions on Biomedical Engineering, vol. 57(5), 2010.

[7] EEG Data set from Epilepsy Center of the University Hospital of Freiburg, http://epilepsy.uni-freiburg.de/freiburg-seizure-prediction-project/eeg-database, visited date: June 10, 2012.

[8] V. Bajaj and R. B. Pachori, "Classification of Seizure and Non-seizure EEG Signals using Empirical Mode Decomposition," IEEE Transactions on Information Technology in Biomedicine, vol. 16(6), Nov 2012.

[9] "Epilepsy data: a few small files (text format)," http://epileptologiebonn.de/cms/front_content.php?idcat=193\&lang=3\&changelang=3, visited date: April 28, 2012.

[10] Y. Song, and J. Zhang," Automatic recognition of epileptic EEG patters via Extreme Learning Machine and multiresolution feature extraction," Elsevier Journal of Expert Systems with Application, vol. 40, 5477-5489, 2013.

[11] J. A. K. Suykens and J. Vandewalle," Least Squares Support Vector Machine Classifiers," Neural Processing Letters, vol. 9(3), 293-300, 1999.

[12] K. D. Brabanter, P. Karsmakers, F. Ojeda, C. Alzate, and J. D. Brabanter, K. Pelckmans, B. De Moor, J. Vandewalle, J.A.K. Suykens, LS-SVMlab Toolbox User's Guide, version 1.8, Katholieke Universiteit Leuven, August 2011.

[13] H. Adeli, Z. Zhou, and N. Dadmehr,"Analysis of EEG records in an epileptic patient using wavelet transform," Journal of Neuroscience Methods, vol. 123, 6987, 2003.

[14] S. Sanei and J.A. Chambers, "EEG Signal Processing," Centre of Digital Signal Processing, Cardiff University, UK, John Wiley \& Sons, Ltd., 2007.

[15] X. Zhang, W. Diao and Z. Cheng, "Wavelet Transform and Singular Value Decomposition of EEG Signal for Pattern Recognition of Complicated Hand Activities," Digital Human Modeling, Lecture Notes in Computer Science, vol. 4561, 294-303, 2007. 